\title{
La télévision sur smartphone : ressorts et implications du renouvellement des modalités d'agrégation
}

Television on smartphone: motives and implications of the renewal of content editing

\section{Virginie Sonet}

\section{OpenEdition Journals}

Édition électronique

URL : https://journals.openedition.org/edc/6167

DOI : 10.4000/edc.6167

ISSN : 2101-0366

Éditeur

Université de Lille

Édition imprimée

Date de publication : 1 juin 2015

Pagination : 47-62

ISBN : 978-2-917562-13-0

ISSN : $1270-6841$

\section{Référence électronique}

Virginie Sonet, «La télévision sur smartphone : ressorts et implications du renouvellement des modalités d'agrégation », Études de communication [En ligne], 44 | 2015, mis en ligne le 01 juin 2015, consulté le 21 septembre 2021. URL : http://journals.openedition.org/edc/6167 ; DOI : https://doi.org/ 10.4000/edc. 6167 


\section{VIRGINIE SONET \\ LA TÉLÉVISION SUR SMARTPHONE : RESSORTS ET IMPLICATIONS DU RENOUVELLEMENT DES MODALITÉS D'AGRÉGATION}

En France, les offres de télévision accessibles en mobilité via les réseaux 3G $(T V 3 G)$, proposées par les opérateurs mobiles se diffusent dès 2006. Ces bouquets ne proposent toutefois les chaînes que dans leur version linéaire et ne permettent pas à celles-ci de commercialiser des espaces publicitaires supplémentaires.

Du côté des chaînes historiques de télévision, cette même période est synonyme de fragilisation économique. Le paysage audiovisuel français s'est considérablement modifié avec le développement de la télévision par ADSL des fournisseurs d'accès à Internet (2003), qui proposent des bouquets de 25 à 60 chaînes et de la TNT (2005) qui offre 25 chaînes généralistes et thématiques gratuitement. La concurrence s'accroît, disperse les audiences et avec elles les revenus publicitaires. En outre, les pratiques audiovisuelles évoluent vers la consommation de contenus délinéarisés (télévision de rattrapage, vidéo à la demande) et de contenus audiovisuels «hors télévision » (vidéos en ligne professionnelles ou amateurs) ${ }^{1}$.

1 En France, en décembre 2010, près de 14 millions d'internautes avaient déjà regardé une vidéo sur YouTube (Comscore, 2010). 
Par ailleurs, les écrans de visionnage se diversifient et se nomadisent (ordinateur, baladeur vidéo, téléphone, tablette), favorisant des consommations en mobilité, facilitées par l'amélioration des réseaux mobiles. À partir de 2009, les smartphones se démocratisent ${ }^{2}$. Les chaînes historiques de télévision sont à la recherche de nouveaux moyens pour accompagner les évolutions des usages, pour rassembler des audiences disséminées et pour gagner en autonomie éditoriale et commerciale par rapport à la TV3G. Elles sont alors incitées à rejoindre ce nouvel écran, notamment sous la forme d'applications. La question se pose alors du devenir des modalités de publication de la télévision sur le smartphone.

En effet, intégrer un nouveau terminal-écran revient à adopter un système technique original. Le produit médiatique délivré est aussi susceptible d'être renouvelé. Or ce sont là deux composantes majeures des dispositifs énonciatifs. Pour Chartron et Rebillard (2004), le «dispositif énonciatif» caractérise les modalités de publication des médias. Il est le résultat de la combinaison de différents éléments que sont le métier d'origine de l'éditeur, les normes et les pratiques professionnelles, la représentation des usages, l'influence du dispositif technique, l'expression d'une singularité éditoriale et la recherche d'une viabilité économique. La logique d'agrégation des contenus traduit donc le métier de l'éditeur et supporte son modèle économique. Une autre dimension peut, selon nous, rejoindre la composition du dispositif énonciatif, celle du modèle de production de l'attention. Comme l'explique Goldhaber (1997), avec la numérisation et la circulation des contenus sur Internet, la surabondance informationnelle et la multiplication des sollicitations ont déplacé la rareté de l'information vers l'attention du consommateur. Boullier (2009) a montré que la manière dont les contenus sont agencés relève de trois modèles de production attentionnelle. D'abord la «fidélisation », qui repose sur la durée de la relation avec le consommateur. La télévision en est un archétype. Ensuite, «l'alerte » relève d'un effet d'agencement collectif. La valeur du service n'est pas le contenu lui-même, mais l'aide à la décision, la recommandation. La notion de réputation de l'émetteur est alors essentielle. Enfin, «l'immersion », dont le jeu vidéo est la figure idéale, s'appuie sur la construction «d'univers persistants »

2 Selon le CREDOC (2013), en France, le taux d'équipement des individus de plus de 12 ans en smartphone passe de $17 \%$ en 2011 à $39 \%$ en 2013. 
(ibid., 240) qui permettent à l'utilisateur de s'immerger dans un monde qu'il contribue lui-même à faire advenir par son action.

Ainsi, dans le domaine de la télévision gratuite et commerciale, le dispositif énonciatif peut être résumé de la manière suivante : la chaîne a pour métier l'édition-diffusion de programmes. La programmation est l'activité qui permet à la chaîne de s'approprier la valeur générée par la vente à un annonceur de l'attention d'une audience anticipée. Son savoir-faire est donc de soutenir les habitudes de visionnage. La production attentionnelle relève alors du modèle de la fidélisation. Cela passe par la structure même de l'agrégation : temporelle, elle est un flux. Dans ce domaine, les chaînes de télévision sont des marques très puissantes. Par ailleurs, le dispositif technique dédié - le téléviseur - ne propose que des chaînes de télévision. Ceci permet à ces dernières d'occuper une place centrale dans la chaîne de valeur : celle de la prescription. En effet, comme le rappellent Benghozi et Paris (2003), dans le domaine de la télévision traditionnelle, les étapes de la programmation et de la diffusion sont rassemblées. Lorsque le téléspectateur choisit un diffuseur, il adhère de facto au choix de programmes de ce dernier.

Dans cet article, nous cherchons donc à comprendre en quoi l'intégration des chaînes de télévision dans un nouveau dispositif technique, en l'occurrence le smartphone, contribue à la configuration d'un dispositif énonciatif inédit.

Pour y répondre, nous mobilisons le matériau de plusieurs enquêtes; une enquête réalisée par entretiens et carnets de bord auprès de 27 utilisateurs de smartphone ("smartphonautes ») ${ }^{3}$ sur leurs pratiques audiovisuelles mobiles (début 2011), une trentaine d'entretiens de professionnels issus des secteurs de la télévision et de la téléphonie mobile (entre 2010 et 2012) et une observation de l'évolution de l'offre des chaînes sur smartphone 4 (de 2010 à 2013).

3 Nous faisons le choix de désigner les utilisateurs de smartphones par le terme «smartphonautes » en référence aux « mobinautes » (personnes qui naviguent sur Internet depuis un téléphone mobile) mais avec la volonté de dépasser cette définition. En effet, notre enquête montre que les utilisateurs de smartphone sont bien plus que des «mobinautes » : ils jouent, lisent, écrivent, écoutent de la musique, travaillent, regardent des vidéos, communiquent, etc. Par ailleurs, ils ne sont «mobispectateurs » (personnes qui regardent des contenus audiovisuels sur un téléphone mobile) qu'au moment où ils mobilisent les fonctions audiovisuelles de leur terminal.

$4 \quad$ Notre périmètre comprend les applications et les comptes de réseaux sociaux de TF1, France Télévisions et M6. Nous retenons aussi Arte et Canal+, 
Nous analysons d'abord le support-écran qu'est le smartphone, ainsi que les formats et les usages qui lui sont associés. Ensuite, au prisme des nouveaux produits médiatiques qu'y proposent les chaînes de télévision, nous examinons quels dispositifs énonciatifs se forment. Pour cela, nous observons le renouvellement des différentes composantes que sont le métier de l'éditeur, l'organisation industrielle, la création de valeur et le ressort attentionnel convoqué. Nous prenons comme observatoire trois modalités inédites d'agrégation : l'application enrichie, l'agrégation communicationnelle et la Social $T V^{5}$. Nous montrons que ces formes ne sont pas de simples déclinaisons, mais un renouvellement du média télévisuel.

\section{Le smartphone, un système technique inédit}

Dans cette première partie, nous examinons les caractéristiques du support qu'est le smartphone. Nous présentons donc dans un premier temps les formats médiatiques et les usages qu'il contribue à favoriser.

Le smartphone est un écran singulier. Au-delà de la mobilité, il est caractérisé par sa polyvalence. Les applications en sont les contenus phares. Leur variété et leur adéquation à ce terminal en favorisent la modularité et en étendent les fonctionnalités. C'est donc sous cette forme que les chaînes de télévision apparaissent en premier lieu sur cet écran. Ces applications sont développées en utilisant les Software Development Kits mis à disposition par les gestionnaires de plateformes mobiles comme Android et Apple. Ces outils de développement, s'ils permettent de proposer des applications ergonomiques, adaptées à l'utilisation en mobilité et sur un petit écran, sont aussi des outils puissants de mise en forme des contenus. En effet, les développeurs sont enjoints à respecter des chartes uniformisantes qui ont pour résultat l'incorporation d'un nombre

dont le modèle économique originel est certes différent (gratuité sans publicité et à péage), mais dont les objectifs assignés aux nouveaux écrans sont très proches de ceux des chaînes gratuites avec publicité.

5 Le CSA (2013) définit la Social TV comme «les technologies apportant un enrichissement des contenus et une interaction entre le téléspectateur et le contenu qu'il regarde ou souhaite regarder et entre les téléspectateurs euxmêmes autour de ce contenu ». Dans ce texte, nous conservons la terminologie proposée par le CSA. 
croissant de fonctions dans les applications, notamment les fonctions de partage.

La possibilité de télécharger une grande variété d'applications élargit donc le champ des possibles pour les smartphonautes. Comme le montrent les études de Médiamétrie (2011, 2012 et 2013), les pratiques les plus populaires sont les pratiques communicationnelles (envoi et réception de SMS, de mails, consultation et alimentation des réseaux socionumériques), informationnelles (consultation de sites d'actualités, d'informations pratiques) et connexionnelles (les pratiques de navigation dans leur ensemble, ainsi que le jeu ${ }^{6}$. Ceci se traduit dans les audiences des applications mobiles; le haut du classement est régulièrement occupé par Google, YouTube, iTunes, Facebook ou Orange.

Notre enquête sur les pratiques audiovisuelles des smartphonautes nous a permis de confirmer que ce troisième écran n'est pas dédié à l'audiovisuel, à la différence du téléviseur et qu'il est un écran fortement communicationnel. Sa mobilité inscrit son utilisation dans une dimension temporelle forte et dans un faisceau de contraintes important ; c'est en effet un écran de la consultation instantanée et opportuniste, qui demande de considérer la disponibilité du réseau mobile et d'un forfait internet adéquat, les co-présents et le contexte d'utilisation.

Nous avons fait émerger quatre registres d'entrée en contact avec les contenus audiovisuels, c'est-à-dire les pratiques en cours des smartphonautes lorsqu'ils visionnent une vidéo. Les registres plus répandus sont l'adressage; la vidéo visionnée a été reçue dans le cadre d'une pratique de communication (mail, SMS, réseaux sociaux) et l'exposition fortuite ; la vidéo a été rencontrée dans le cadre d'une pratique de connexion (navigation, lecture d'information). Puis, vient la recherche opportuniste ; le contenu a été sciemment recherché dans un usage relationnel (le montrer à des amis) ou phonique (« écouter » un clip). Enfin, en mineur, nous trouvons la préméditation; le visionnage de la vidéo est prévu. Souvent, la vidéo a été téléchargée en amont dans le smartphone pour être visible hors connexion.

6 À titre d'exemple, $80 \%$ des smartphonautes interrogés déclarent ne pas pouvoir se passer des SMS/MMS, $56 \%$ d'Internet et des e-mails et $25 \%$ des réseaux sociaux (Médiamétrie, 2012, « Jamais sans mon smartphone. Tous geeks ? », AudienceMag). 
Les deux registres majoritaires, l'adressage et l'exposition fortuite, relèvent donc de pratiques de communication et de connexion.

Cela a au moins deux conséquences sur le dispositif énonciatif originel des chaînes de télévision. Premièrement, des applications non audiovisuelles sont en mesure de proposer elles aussi des contenus vidéos et donc entrent en concurrence avec les applications des éditeurs audiovisuels traditionnels. Deuxièmement, le fait que les registres d'entrée en contact avec les contenus audiovisuels relèvent de pratiques largement communicationnelles et connexionnelles contribue à modifier les logiques de consommation. En effet, celles qui se construisent sur l'écran du smartphone font perdre aux chaînes la fonction centrale des modèles socio-économiques des médias de diffusion: la prescription de la consommation des programmes. Ainsi, dans l'économie de l'attention qui caractérise les industries médiatiques, tous ces éléments se traduisent sur cet écran par au moins deux répercussions que nous retenons pour notre propos. D'un côté, la concurrence pour l'attention de l'utilisateur est considérable. Les multiples applications à sa disposition concourent toutes (ou presque) pour son utilisation et pour les budgets publicitaires des annonceurs. De l'autre, la polyvalence du smartphone et la diversité des pratiques qui s'y développent remettent en cause les modalités de consommations audiovisuelles, en tant que composantes du dispositif énonciatif de la télévision traditionnelle.

Ainsi, l'intégration du smartphone par les chaînes de télévision marque une certaine rupture. Cet écran n'est pas exclusivement alimenté par des diffuseurs, les modalités de consommation de l'audiovisuel sont très éloignées de celles de la télévision, la fonction de prescription de la consommation audiovisuelle est complexifiée et les formats de publication sont fortement contraints par les systèmes techniques - autant de composantes renouvelées du dispositif éditorial. Les chaînes doivent donc rénover les modalités de leur publication mais aussi leurs moyens de capter et de valoriser l'attention des utilisateurs.

\section{Trois modalités inédites de publication et leurs implications}

Nous examinons à présent les ressorts et les implications des trois grands types de publication adoptés par les chaînes sur le 
smartphone : l'application enrichie, l'agrégation communicationnelle et la Social TV.

\section{L'application enrichie comme carrefour de pratiques}

Les applications mobiles des chaînes de télévision ont pour objectif de rassembler de nouvelles audiences, de permettre la réalisation d'économies d'échelle autour des programmes et de proposer aux annonceurs des espaces publicitaires multi-écrans.

Kessous (2011) explique que lorsque des firmes entrent en concurrence pour capter l'attention des consommateurs, elles doivent émettre plus de signaux, fournir encore plus d'informations et plus variées. Nous avons relevé l'ensemble des contenus et des services rencontrés de manière plus ou moins systématique dans les applications des chaînes de télévision. Tout d'abord, parmi les contenus audiovisuels proposés, nous trouvons le direct, des programmes en rattrapage, des extraits et des bonus associés à certaines émissions. Nous notons aussi la présence de contenus textuels qui accompagnent certaines vidéos. Ensuite, les applications proposent couramment des services comme le guide des programmes, des liens vers d'autres applications ou des sites partenaires, des jeux et des dispositifs (que nous développons plus bas) permettant différentes formes d'interactivité avec un programme en cours de diffusion. Nous notons également le développement des outils améliorant le confort de la navigation et du visionnage, comme la gestion des favoris, les alertes personnalisées, le contrôle du direct, le moteur de recherche interne ou encore la géolocalisation et le contrôle parental. Enfin, dans l'ensemble des applications observées, nous avons constaté l'intégration des fonctions de partage associées à Facebook, Twitter mais aussi aux mails et aux SMS.

La conception de ces applications repose sur la récurrence de leur consultation et traduit une volonté de reconquérir l'attention des utilisateurs sur le régime de la fidélisation. Ce choix éditorial consiste à agréger plusieurs types de services parmi ceux qui rassemblent le plus d'audience sur les smartphones, dans une sorte de portail. Les chaînes procèdent à une nouvelle forme d'agrégation non plus temporelle, mais connexionnelle, au sens des pratiques des smartphonautes, mêlant navigation et communication. Celle-ci exige d'assembler «autre chose» que des programmes dans une grille. 
Non seulement, l'agrégation de ces multiples services et contenus contraint les chaînes à engager de nouveaux coûts et de nouvelles compétences mais elle les prive également de la fonction qui leur permet traditionnellement de s'approprier de la valeur. En effet, de programmateurs-diffuseurs, prescriptrices de la consommation de programmes, elles migrent vers le métier d'éditeur d'applications enrichies. La tentation de se constituer en carrefour des diverses pratiques sur les smartphones apparait vaine puisque dans ce métier, elles ne font pas référence. Les chaînes ne sont pas des marques fortes et mondiales associées à ce type de pratiques. Les applications «portails» les plus fréquentées reposent sur un service originel puissant, incitant à une connexion répétée : le mail, la messagerie instantanée, le moteur de recherche. Ce qui n'est pas le cas des chaînes de télévision. De surcroît, les audiences de ces applications restent limitées, aléatoires et méconnues. Elles faillissent donc ici à exploiter le régime attentionnel de la fidélisation.

Pour ce nouveau média, la logique de distribution et de consommation relève du modèle éditorial où les contenus sont délivrés à la pièce. Or les chaînes lui appliquent un modèle d'affaires fondé sur l'audience et la gratuité, hérité d'une agrégation temporelle de flot. Ainsi, cette logique de publication dépasse la seule dimension des formats pour atteindre l'ensemble du dispositif énonciatif, y compris l'organisation industrielle et les logiques économiques.

\section{L'agrégation communicationnelle}

Parallèlement, les usages continuent d'évoluer et la consultation des réseaux socionumériques (RSN) depuis les smartphones ne cesse d'augmenter. À la fin 2012, $25 \%$ des smartphonautes jugent ne plus pouvoir s'en passer en mobilité (Médiamétrie, 2012). Comme l'a montré notre enquête auprès des utilisateurs, les RSN sont aussi devenus des espaces de consommation de contenus audiovisuels? Ce sont des signaux forts pour les chaînes de télévision. Elles décident alors de faire de leurs contenus l'objet de ces pratiques communicationnelles, fortement prescriptrices de consommation

$7 \quad$ Facebook est le deuxième site après YouTube de visionnage de vidéos. En mai 2013, aux Etats-Unis, près de 730 millions de vidéos ont été visionnées sur Facebook (nous ne disposons pas des données pour la France) (Comscore, 2013). 
audiovisuelle. Leur objectif est de recruter de nouvelles audiences en encourageant la circulation des contenus et en s'appuyant sur la recommandation interpersonnelle. Elles tentent ainsi de mettre à contribution ces pratiques dans la promotion de leurs programmes.

Nous avons relevé deux grands axes de mise en œuvre : d'un côté, les chaînes créent leur propre compte dans les réseaux sociaux les plus populaires (Facebook et Twitter). Elles multiplient les publications de statuts, de contenus et de tweets pour apparaître fréquemment dans les flux d'actualité des utilisateurs ${ }^{8}$. De l'autre, elles incorporent les fonctions de partage au sein même de leur application. Chaque programme peut dès lors être transféré par le smartphonaute vers ses interlocuteurs, sous la forme d'un lien hypertexte, par mail, par SMS ou partagé sur les RSN.

Cette modalité d'agrégation vise donc à exploiter les pratiques communicationnelles des smartphonautes. Quel dispositif énonciatif se met alors en place?

Tout d'abord, la logique de production de l'attention n'est plus la fidélisation mais l'alerte, inédite pour les chaînes. Dans ce régime, le contenu n'est pas valorisé pour lui-même, mais parce qu'il est adressé par un membre du réseau social de l'utilisateur (professionnel, amical, familial). Cette personne agit alors comme un réducteur d'incertitude pour le programme : en le relayant personnellement, elle lui transfère sa propre réputation. Les récepteurs peuvent ainsi être plus enclins à visionner le programme. Ces pratiques correspondent particulièrement aux registres de l'adressage et de l'exposition fortuite où le contenu audiovisuel est reçu ou rencontré. Mais ce contenu est aussi en quelque sorte imposé au smartphonaute. En effet, dans le cadre des RSN, le contenu est intégré dans le fil d'actualités de Facebook ou la «Time Line » de Twitter. L'utilisateur a certes la liberté de le visionner ou non mais il n'a pas le choix du contenu luimême. La fonction de prescription est donc déplacée de la chaîne vers l'utilisateur, via le flux du RSN.

Ensuite, en proposant leurs contenus dans les RSN, les chaînes s'inscrivent dans une nouvelle organisation industrielle. En effet, ces réseaux sont des «infomédiaires sociaux », i.e. des plateformes

$8 \quad$ Toutes les grandes chaînes ont créé des comptes sur Facebook et sur Twitter entre fin 2008 et 2011. En 2013, TF1 compte 1,7 million d'abonnés sur Facebook et 616000 sur Twitter, France 2 respectivement 318000 et 380000 . TF1 publie en moyenne 13 tweets quotidiens, France 2, 9,5. 
capables de mettre en contact une offre hétérogène et pléthorique d'informations avec une demande éclatée de la part de publics diversifiés. Selon Rieder et Smyrnaios (2012), ils sont susceptibles de générer du trafic et d'accroître l'audience, non pas à travers un algorithme (comme Google News), mais via l'interaction entre usagers à travers des plateformes. Toutefois, comme le précisent les auteurs, l'infomédiation sociale, bien que source d'audience pour les sites, n'apporte encore qu'un faible trafic ${ }^{9}$. En outre, Beauvisage et al. (2011) montrent que la viralité et la contagion sont des «figures idéales» de la diffusion des contenus sur le web. Il est en effet difficile de les identifier objectivement en raison de nombreuses variables qui influent sur la transmission.

Enfin, dans les RSN, les chaînes de télévision deviennent des animateurs de pages dont elles n'ont pas la maîtrise éditoriale. Non seulement le format des statuts publiés est imposé par la plateforme, mais les espaces connexes aux publications restent la propriété du RSN qui y incruste ses propres encarts publicitaires. Une chaîne n'a donc pas la possibilité de commercialiser des espaces publicitaires sur sa propre page Facebook. Elle doit donc embarquer la publicité au sein même de ses contenus (sous la forme de spots en pre-roll notamment). Les chaînes de télévision inscrivent donc leurs contenus et leur marque dans cet autre flux contrôlé par les RSN qui en récupèrent la valeur. En effet, ils conservent les données personnelles des utilisateurs ainsi que les recettes publicitaires. Il s'agit donc d'une situation de coopétition économique. Il y a coopération entre le RSN, apporteur d'audience, et les éditeurs, apporteurs de contenus. Il y a aussi compétition entre les deux acteurs pour le budget des annonceurs, et celle-ci est défavorable aux chaînes de télévision qui n'y détiennent pas d'espaces publicitaires propres.

Face à ces écueils, les chaînes se tournent vers une forme alternative de présence sur le smartphone : les dispositifs interactifs de Social TV. Le smartphone est alors mobilisé comme un écran annexe qui accompagne le visionnage du programme sur le téléviseur.

9 «Sur un échantillon de douze sites de presse français, l'apport moyen de Twitter en trafic ne dépasse pas les 0,5\%» (Rieder et Smyrnaios, 2012, 214). 


\section{Le smartphone comme second écran : l'interaction en perspective}

Wolton, Missika (1983) et Mehl (2002) ont montré depuis longtemps l'existence des conversations autour de la télévision. Selon Médiamétrie ces conversations seraient actuellement amplifiées par deux facteurs. D'un côté, l'utilisation simultanée d'écrans va croissant, le visionnage de la télévision s'accompagne d'une autre activité sur un écran différent, en particulier sur le smartphone ${ }^{10}$. De l'autre, les discussions «peuvent aujourd'hui s'épanouir sur les réseaux sociaux », où les échanges autour des programmes « ont lieu de manière synchrone » (Médiamétrie, 2013). En 2012, un tweet sur deux, soit plusieurs centaines de milliers de tweets par semaine, aurait pour sujet la télévision (CSA, 2013). Les chaînes conçoivent dès lors le smartphone comme un «compagnon » susceptible de diriger de nouveau l'attention de l'utilisateur sur le téléviseur qui demeure l'écran le plus valorisé11. Il s'agit ainsi de revaloriser les programmes et la diffusion en occupant simultanément le téléspectateur sur son smartphone pour éviter la dispersion de son attention. Les chaînes convoquent ainsi le caractère interactif du smartphone et exploitent les usages qui lui sont rattachés.

Il existe plusieurs déclinaisons de la Social TV. Tout d'abord, les dispositifs élémentaires consistent à encourager les téléspectateurs à commenter les programmes sur les réseaux sociaux, depuis leur smartphone, à travers un hashtag (\#) ${ }^{12}$. Originellement proposés spontanément par les utilisateurs, ces hashtags sont de plus en plus préemptés par les chaînes. Certains se formalisent, comme \#NMA13, pour les NRJ Music Awards 2013, voire structurent le nom de l'émission (la matinale de Canal+, présentée par Bruce Toussaint, a pour nom \#TeamToussaint).

Ensuite, des dispositifs plus élaborés émergent à travers lesquels, les chaînes visent le régime attentionnel très convoité de l'immersion. Les chaînes combinent alors l'événement télévisuel

10 En 2013, deux personnes sur trois utilisent au moins une fois par semaine un autre écran devant leur télévision (Médiamétrie, 2013).

11 De 2009 à 2013, en ce qui concerne le chiffre d'affaires publicitaire, la télévision reste le premier média ( $30 \%$ en 2009 et $37 \%$ en 2013), devant Internet (16\% en 2009 et $10 \%$ en 2013) (CSA, 2014).

12 Nous empruntons la description du hashtag à Jeanne-Perrier (2010, 133) : "Le hashtag correspond au signe suivant: \#. Ce signe, suivi d'un mot, permet de retrouver tous les tweets qui sont en rapport avec le terme choisi ». 
(un programme fédérateur d'audience en direct), la conversation synchrone sur les RSN, le jeu, la participation ou le pronostic et l'activation des communautés de fans. Elles conçoivent des univers persistants caractéristiques de l'immersion où l'engagement est présumé important (Boullier, 2009). Ainsi, avant la diffusion le dispositif promeut via les RSN le programme et constitue une première communauté de fans. Pendant la diffusion, le second écran est mobilisé au profit du programme. Il maintient l'audience et son attention en incitant à l'interaction avec le programme diffusé, par exemple à travers l'affichage à l'antenne des meilleurs tweets des téléspectateurs. À l'issue de la diffusion, l'intérêt pour le programme est maintenu via les RSN, au sein de la communauté des fans, en vue d'une saison ultérieure. Le télé-crochet The Voice, sur TF1 en est un archétype.

Ces dispositifs complexes tiennent à la capacité que conservent les grandes chaînes à créer des événements télévisuels fédérateurs. Comme le relève Fonnet $(2010,206)$ à propos de la programmation de rencontres sportives, pour "faire événement », il est nécessaire de rassembler au moins deux des composantes suivantes : " un drapeau, un champion, un enjeu ». Les programmes qui se prêtent le mieux à ce type de dispositifs sont donc le sport, les cérémonies culturelles et artistiques (comme les Césars ou les NRJ Music Awards), les débats électoraux et la télé-réalité ${ }^{13}$.

La Social TV peut favoriser le visionnage du programme luimême (en direct ou en rattrapage) dans des espaces monétisés par la publicité (téléviseur, site internet, application), mais elle s'avère coûteuse. Le développement des plateformes, l'animation, les opérations événementielles, la création d'une application dédiée, la souscription à une solution de mesure des audiences sociales, atteignent plusieurs centaines de milliers d'euros (CSA, 2013). De surcroît, le lien entre l'audience sociale d'un programme, i.e. l'activité qu'il suscite sur les RSN, et la réalisation effective d'audiences télévisuelles n'est pas encore solidement établi. Médiamétrie, malgré son alliance avec Mesagraph autour d'un outil d'analyse du lien statistique entre la conversation numérique et l'audience, n'a, pour l'heure, formulé aucun résultat montrant objectivement ce lien (Médiamétrie, 2013).

13 Ce sont Secret Story, avec près de 900000 tweets, le tennis et le football qui occupent le haut du classement des « audiences de la Social TV» (Mesagraph, 2014). 
Néanmoins, avec la Social TV, le smartphone n'est pas conçu comme le support de visionnage des contenus audiovisuels et le téléviseur est remis au centre du modèle. C'est donc dans ce cadre que le dispositif énonciatif originel de la télévision est le moins déséquilibré.

\section{Conclusion}

Nous avons vu que pour tenter de ré-agréger des audiences disséminées par l'accroissement de l'offre audiovisuelle et pour suivre le développement des usages, les chaînes de télévision se déploient sur l'écran du smartphone. Ce système technique favorise des formats de publication inédits (applications, réseaux sociaux mobiles) ainsi que des usages hybrides, où le visionnage de contenus audiovisuels s'inscrit largement dans des pratiques de communication et de connexion. Dès lors, les modalités de publication adoptées par les chaînes de télévision sur ce nouvel écran participent de la construction de dispositifs énonciatifs originaux. Les chaînes voient en effet leur métier modifié (elles deviennent éditrices d'applications et animatrices de comptes sur les réseaux socionumériques), leur fonction de prescription amoindrie et leur modèle économique originel fragilisé.

L'application enrichie brigue une fréquentation massive et fidèle des utilisateurs. L'agrégation communicationnelle tend à faire des pratiques communicationnelles le support de la promotion et de la diffusion des programmes. Toutes deux renouvellent profondément le dispositif énonciatif originel de la télévision en sa défaveur. Ces nouvelles mises en écran ne sont donc pas de simples déclinaisons pour les chaînes, ce sont de nouveaux formats d'édition et de publication. Ils exigent d'agréger et de présenter leurs contenus et leurs services dans une forme imposée par des dispositifs socio-technoéconomiques puissants. Ils participent en outre d'une consommation et de modèles économiques spécifiques, au sein desquels les chaînes perdent le contrôle de la valeur qu'elles contribuent à créer. L'hérédité de leur modèle socio-économique traditionnel et leur moindre maîtrise des nouveaux systèmes techniques expliquent en partie la fragilité des comportements stratégiques des chaînes. Seule la Social TV, en remettant le téléviseur au centre de la stratégie, permet pour l'heure le maintien des composantes du dispositif énonciatif d'origine. En effet, 
l'interaction proposée par les chaînes avec le téléspectateur demeure dans une visée marketing et ne remet pas en question leur statut de diffuseur. Leur modèle économique n'est donc pas renouvelé en profondeur.

Plus qu'un troisième écran de diffusion des programmes, le smartphone se dévoile donc comme un tiers écran. Les formes qui s'y construisent, ainsi que leurs implications, sont révélatrices d'une crise médiatique. Pour Altman $(2000,13)$, une telle crise comporterait trois éléments :

Identité multiple, lutte juridictionnelle et solution surdéterminée qui se chevauchent plutôt que de se suivre comme des étapes distinctes. La crise étant provoquée par une nouvelle technologie, une nouvelle situation sociale, voire un nouveau système d'exposition, l'identité du média est à tel point mise en question que ce média en vient à s'apparenter à plusieurs médias existants.

Nous serions donc dans un moment d'intermédialité, au sens retenu par l'auteur, c'est-à-dire moins un simple mélange de médias qu'une période "pendant laquelle une forme destinée à devenir un média à part entière se trouve encore à tel point tiraillée entre plusieurs médias que son identité reste en suspens » (ibid., 11). Notre travail nous permet ainsi de corroborer les termes de Bardini et $a l .(2000,180)$ signalant que la première intermédialité qu'ils ont observée est « celle des producteurs appliquant à un nouveau média la même logique que le média d'origine ».

VIRGINIE SONET IFP-CARISM Université Paris II Panthéon-Assas 


\section{BIBLIOGRAPHIE}

Altman R., (2000), Technologie et textualité de l'intermédialité, in La croisée des médias, Sociétés et Représentations, n 9, CREDHESS, pp. 11-20.

Bardini T. et al., (2000), Des nouvelles de l'interacteur : phénomène de convergence entre la télévision et Internet, in Société et représentations, Paris, CREDHESS, pp. 161-180.

Beauvisage T. et al., (2011), Le succès sur Internet repose-t-il sur la contagion? Une analyse des recherches sur la viralité, in Tracés, ${ }^{\circ} 21$, pp. 151-166.

Benghozi P.-J. et Paris T., (2003), De l'intermédiation à la prescription : le cas de la télévision, in Revue française de gestion, $\mathrm{n}^{\circ}$ 142, pp. 205-227.

Boullier D., (2009), Les industries de l'attention : fidélisation, alerte ou immersion, in Réseaux, $\mathrm{n}^{\circ} 154$, pp. 231-246.

Chartron G. et Rebillard F., (2004), Modèles de publication sur le web, Rapport d'activité dans le cadre de l'AS-CNRS 103, https://halshs.archives-ouvertes.fr/ sic_00001159/document, date de la dernière visite : le 14 janvier 2015.

Comscore, (2010), The 2010 Europe Digital Year in Review, http://www.comscore. com/fre/Insights/Presentations-and-Whitepapers/2011/2010-Europe-Digital-Year-inReview, date de la dernière visite : le 14 janvier 2015.

Comscore, (2013), U.S. Online Video Rankings, http://www.comscore.com/fre/ Insights/Press-Releases/2013/6/comScore-Releases-May-2013-US-Online-VideoRankings, date de la dernière visite : le 14 janvier 2015.

CREDOC, (2013), La diffusion des technologies de l'information et de la communication dans la société française, collection des rapports, $\mathrm{n}^{\circ} 297$, novembre 2013.

CSA, (2013), Première approche de la télévision sociale, Commission de réflexion prospective sur l'audiovisuel, http://www.csa.fr/Etudes-et-publications/Lesetudes/Les-etudes-du-CSA/Premiere-approche-de-la-television-sociale, date de la dernière visite : le 14 janvier 2015.

CSA, (2014), Les chiffres clés de l'audiovisuel, édition du $1^{\text {er }}$ semestre 2014, en ligne : http://www.csa.fr/Etudes-et-publications/Les-chiffres-cles/Les-chiffrescles-de-1-audiovisuel-francais-Edition-du-1er-semestre-2014, date de la dernière visite : le 14 janvier 2015.

Fonnet L., (2010), La programmation de la télévision à l'ère numérique, Paris, Dixit, $303 \mathrm{p}$.

Goldhaber M.-H., (1997), The attention economy and the net, in First Monday, vol. 2, $\mathrm{n}^{\circ} 4$, http://firstmonday.org/ojs/index.php/fm/article/view/519/440, date de la dernière visite : le 14 janvier 2015.

Jeanne-Perrier V., (2010), Parler de la télévision sur Twitter : une "réception» oblique à partir d'une « conversation » médiatique ?, in Communication \& langage, $\mathrm{n}^{\circ} 156$, pp. 127-147.

Kessous E., (2011), L'économie de l'attention et le marketing des traces, Actes du colloque «Web social, communautés virtuelles et consommation », 79e congrès international ACFAS, Sherbrooke, 11 mai 2011, http://manuel.boutet.free.fr/ Kessous2011attention.pdf, date de la dernière visite : le 14 janvier 2015. 
Médiamétrie, (2012), Jamais sans mon smartphone. Tous geeks ?, AudienceMag, 12/12/2012, http://www.audiencelemag.com/?article=48, date de la dernière visite : le 14 janvier 2015.

Médiamétrie, (2013), Médiamétrie lance l'étude Social TV, communiqué de presse diffusé le 04/07/13.

Médiamétrie, (2011, 2012, 2013), Audience des applications mobiles en France, communiqués de presse, communiqués de presse diffusés les 28/07/11, 13/08/12 et 07/08/13.

Mehl D., (2002), La télévision relationnelle, in Cahiers internationaux de sociologie, $\mathrm{n}^{\circ} 112$, pp. 63-95.

Mesagraph, (2014), Mesagraph dévoile le classement des émissions TV les plus populaires sur Twitter en 2013, http://blog.mesagraph.com/tag/2013/, date de la dernière visite : le 14 janvier 2015.

Rieder B. et Smyrnaios N., (2012), Pluralisme et infomédiation sociale de l'actualité : le cas de Twitter, in Réseaux, $\mathrm{n}^{\circ} 176$, pp. 105-139.

Wolton D. et Missika J.-L., (1983), La folle du logis, la télévision dans les sociétés démocratiques, Paris, Gallimard, 348 p. 